\title{
Etude théorique et expérimentale du transistor à effet de champ à hétérojonction (*)
}

\author{
A. Cappy, A. Vanoverschelde, J. Zimmermann, P. Philippe, C. Versnaeyen et G. Salmer \\ Centre Hyperfréquences et Semiconducteurs (**), U.E.R. d'I.E.E.A., Bât. P4, \\ Université des Sciences et Techniques de Lille I, 59655 Villeneuve d'Ascq Cedex, France
}

(Reçu le 25 juillet 1983, accepté le ler août 1983)

\begin{abstract}
Résumé. - La dynamique des électrons dans un gaz bidimensionnel est étudiée par la méthode de Monte-Carlo. Les hétérojonctions envisagées sont $\mathrm{GaAs} / \mathrm{GaAlAs}$ et $\mathrm{GaInAs} / \mathrm{InP}$. Ces résultats sont alors utilisés pour une modélisation du transistor à hétérojonction (TEGFET). Une comparaison de cette structure avec le TEC conventionnel est effectuée dans les deux applications suivantes : l'amplification faible bruit et les circuits logiques à couplage direct. Une supériorité intrinsèque du TEGFET dans chacune de ces applications est mise en évidence.
\end{abstract}

Abstract. - The electron dynamics in a two dimensional electron gas is studied by the Monte-Carlo procedure. $\mathrm{GaAs} / \mathrm{GaAlAs}$ and $\mathrm{GaInAs} / \mathrm{InP}$ heterojunctions are considered. The results of these simulations are then used in a theoretical TEGFET model. A comparison between TEGFET and conventional FET is carried out for two possible applications : low noise amplifier and DCFL circuits. An intrinsic superiority of TEGFET is pointed out.

\section{Introduction.}

Depuis environ trois années, de nombreux travaux ont montré que la couche bidimensionnelle d'électrons accumulés à l'interface d'une hétérojonction pouvait être utilisée avec profit comme couche active d'un transistor à effet de champ [1,3]. Néanmoins, il n'existe à ce jour que des modèles analytiques ou quasi analytiques [4] de cette structure; les limitations fondamentales des performances sont donc assez mal connues.

Le but de cet article est d'étudier ces limitations et de les comparer avec celles du TEC classique. A cette fin, nous étudions dans une première partie, les caractéristiques de la dynamique des électrons dans la couche d'accumulation. Les résultats de cette étude sont alors introduits dans un modèle du TEGFET qui sera décrit dans la deuxième partie de cet article. Enfin, les performances de cette structure seront comparées avec celles du TEC classique.

\section{Modélisation microscopique.}

La simulation microscopique bidimensionnelle du transistor à effet de champ à hétérojonction est particulièrement difficile étant donné les dimensions géométriques de cette structure et la concentration en

(*) Travail effectué avec le soutien de la D.R.E.T. (contrat $\left.n^{\circ} 81 / 611\right)$.

(**) L.A.-CNRS no 287. impuretés élevée de la couche d'AlGaAs. Néanmoins, l'étude de la dynamique électronique y est fondamentale car elle permet de différencier le transistor à effet de champ classique du TEGFET.

Notre modèle, schématisé sur la figure 1 considère un plan de section normal à la grille du transistor. Il repose sur une résolution simultanée de l'équation de Boltzmann par la méthode de Monte-Carlo et de
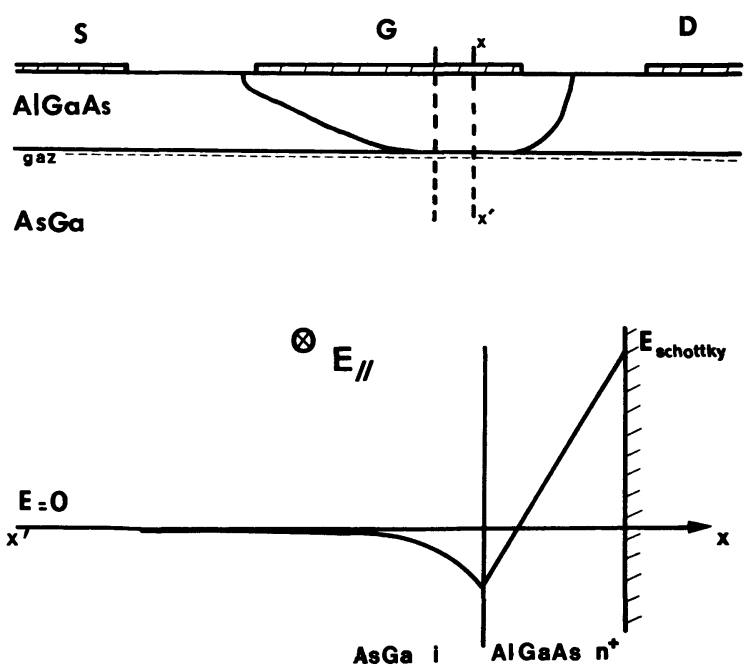

Fig. 1. - Coupe transversale du transistor TEGFET et configuration du champ électrique.

[TEGFET transverse section and electric field.] 
l'équation de Poisson unidimensionnelle. Les conditions aux limites sont fixées par le champ électrique sur la grille et dans la couche tampon $\left(E_{\perp}=0\right)$. L'influence de la tension drain-source sera simulée par l'application d'un champ parallèle $E_{\|}$.

Les conditions de passage d'un électron à travers l'hétérojonction sont régies par les règles suivantes :

- le transfert dans l'espace réel ne pourra s'effectuer que si l'énergie portée par la composante de vitesse perpendiculaire à l'interface est supérieure à la hauteur de barrière $\Delta E_{\mathrm{c}}$. Dans ce cas, il y a conservation du moment parallèle à l'hétérojonction et conservation de l'énergie totale. De plus, on supposera que le franchissement de l'hétérojonction se fait entre vallées équivalentes;

- si l'énergie de l'électron est par contre insuffisante, celui-ci subit alors à l'interface une réflexion de type spéculaire.

Le comptage des électrons dans chaque case $x_{i}$ au cours de la simulation et l'intégration de l'équation de Poisson permettent de déterminer la densité volumique des charges mobiles et la distribution du champ électrique dans la structure. Un résultat typique est donné sur la figure 2 où le champ électrique sur le contact Schottky est fixé à $250 \mathrm{kV} / \mathrm{cm}$. On peut constater tout d'abord que la couche d'AlGaAs est, dans le cas d'un champ parallèle faible, totalement vide de charges mobiles. Celles-ci s'accumulent près de l'interface dans l'arséniure de gallium non dopé et conduisent à une concentration superficielle voisine de $2,5 \times 10^{15} \mathrm{~m}^{-2}$.

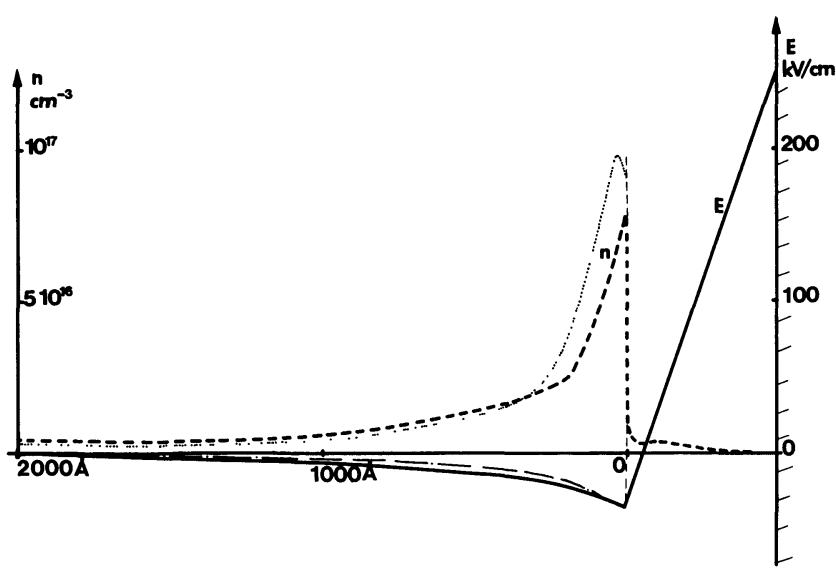

Fig. 2. - Distribution des charges mobiles et champ électrique à l'aplomb de la grille du TEGFET. $E_{\|}=1 \mathrm{kV} / \mathrm{cm}$ : (.......) $\quad n(x),(-\cdot-) \quad E(x) ; \quad E_{\|}=10 \mathrm{kV} / \mathrm{cm}:(---)^{--)}$ $n(x),\left(\stackrel{(\longrightarrow)}{\longrightarrow} E(x) . N_{\mathrm{DAIGaAs}}=4 \times 10^{17} \mathrm{~cm}^{-3}, N_{\mathrm{D} \mathrm{ASGa}}=\right.$ $10^{14} \mathrm{~cm}^{-3}$.

\section{[Carrier density and electric field in the TEGFET's.]}

L'influence de la tension drain-source du transistor traduite par l'application du champ électrique parallèle à l'interface se manifeste par un phénomène de diffusion de la charge d'accumulation dans la couche tampon et par un transfert des électrons dans leur matériau d'origine aux champs les plus élevés. Ces deux phénomènes s'expliquent par une augmentation de l'énergie moyenne des porteurs. Ainsi, ils diffusent plus facilement dans l'AsGa du fait de l'augmentation de la longueur de Debye avec l'énergie. D'autre part, la possibilité de franchir la barrière de potentiel à l'interface est alors augmentée.

Par sommation sur tous les électrons composant la simulation, on peut obtenir la vitesse moyenne et l'énergie moyenne dans la structure en fonction du champ $E_{\|}$(Fig. 3). On constate qu'il y a conservation de la valeur de la mobilité électronique en champ faible de l'AsGa intrinsèque mais que la vitesse de saturation est notablement diminuée ceci en raison du transfert des électrons dans la couche $\mathrm{AlGaAs}$ de vitesse de saturation plus faible.
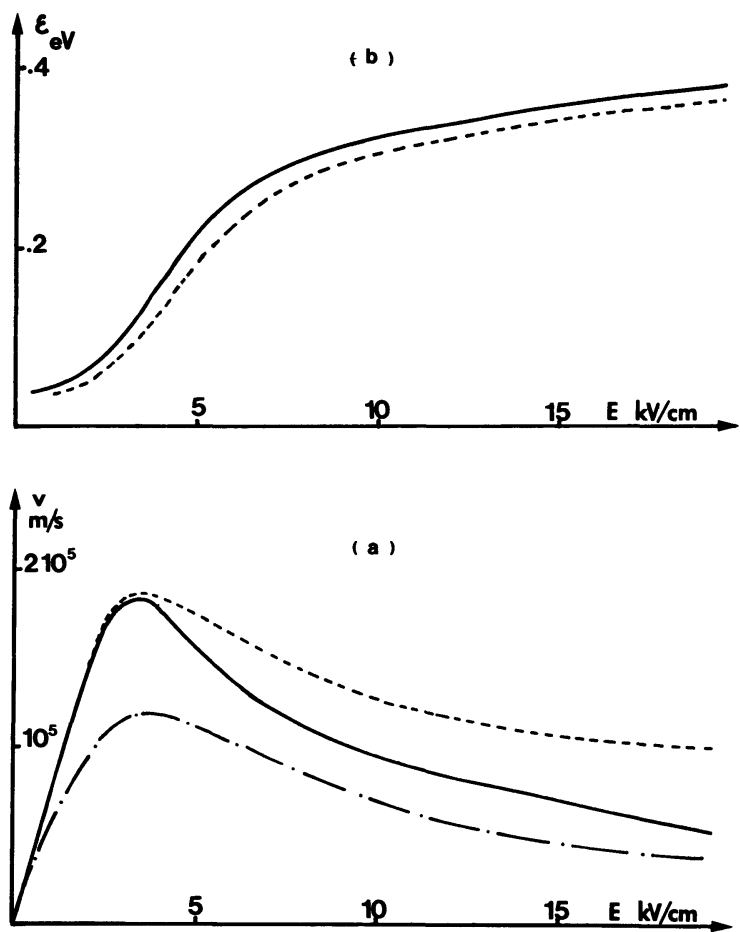

Fig. 3. - Vitesse moyenne (a) et énergie moyenne $(b)$ des électrons en fonction du champ parallèle (-) TEGFET GaAs $/$ GaAlAs : $E_{\text {grille }}=250 \mathrm{kV} / \mathrm{cm}$; (—. - TEGFET $\mathrm{GaAs} / \mathrm{GaAlAs}: E_{\text {grille }}=180 \mathrm{kV} / \mathrm{cm}$; (-------) GaAs intrinsèque.

[Average velocity and energy versus parallel field $E_{\|}$.]

Une étude prospective similaire a été effectuée à partir des matériaux GaInAs et InP. L'influence du champ parallèle se manifeste de la même manière que précédemment sur la charge accumulée à l'interface (Fig. 4). Mais, pour des valeurs de $E_{\|}$supérieures au champ de seuil, la vitesse contrairement au cas de l'hétérojonction $\mathrm{GaAs} / \mathrm{GaAlAs}$, devient supérieure à celle du matériau non dopé (Fig. 5). Ceci peut s'expliquer par la contribution des électrons retransférés dans le matériau InP, dont la vitesse pour ces valeurs de champ est supérieure à celle du GaInAs. 


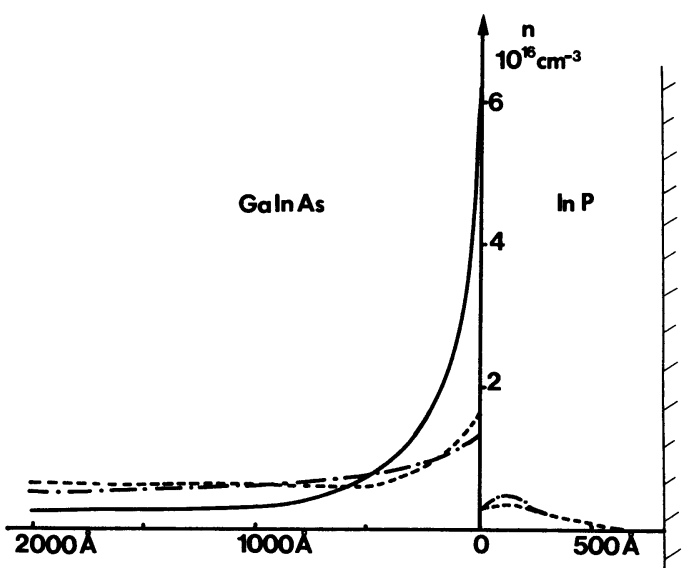

Fig. 4. - Répartition des électrons dans l'hétérostructure GaInAs/InP $E_{\text {grille }}=100 \mathrm{kV} / \mathrm{cm},(\longrightarrow) E_{\|}=0 ;(--)$ $E_{\|}=5 \mathrm{kV} / \mathrm{cm} ;(---)_{\|}=15 \mathrm{kV} / \mathrm{cm} ; N_{\mathrm{DInP}}=10^{17} \mathrm{~cm}^{-3}$, $N_{\text {DGaInAs }}=10^{15} \mathrm{~cm}^{-3}$.

[Carrier density in the GaInAs/InP heterostructure.]

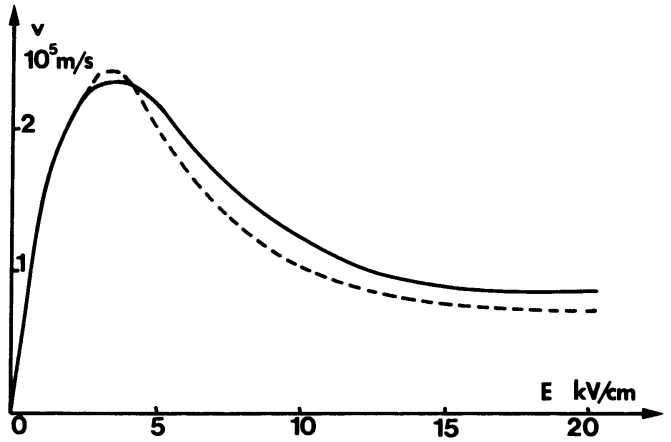

Fig. 5. - Vitesse électronique moyenne en fonction du champ parallèle. (—) TEGFET GaInAs/InP, $E_{\text {grille }}=$ $100 \mathrm{kV} / \mathrm{cm}$; (------) GaInAs intrinsèque.

[Average velocity versus parallel field.]

Cette étude est complétée par l'analyse de l'influence du champ électrique de grille du transistor. On constate sur la figure 6 qu'il y a commande de la charge d'accumulation par la tension $V_{\mathrm{gg}}$. En effet, pour des tensions suffisantes telles que la couche d'AlGaAs soit désertée, l'application d'une tension supplémentaire entraîne une variation linéaire du champ à l'interface et donc une variation de la charge superficielle $n_{\mathrm{s}}$. Cette commande est sensiblement linéaire comme le montre la figure 7 au moins tant que l'apport énergétique dû au champ parallèle n'est pas suffisant pour permettre le franchissement de l'hétérojonction. Comme précédemment, une étude de la vitesse électronique dans le dispositif a été effectuée pour une polarisation de grille plus faible. On constate que la vitesse limite (Fig. 3) diminue en raison d'un confinement moins strict des électrons dans le matériau AsGa.

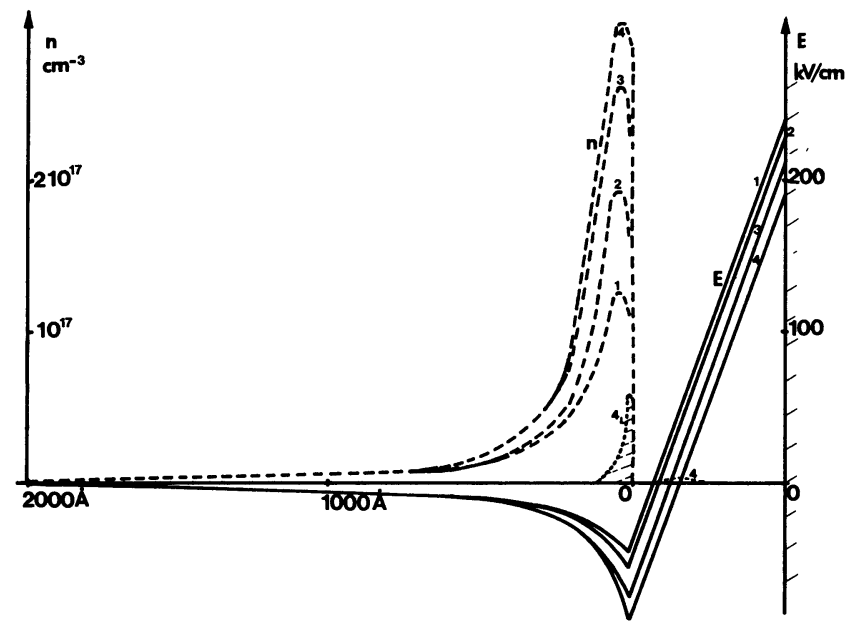

Fig. 6. - Répartition des électrons et du champ électrique dans le TEGFET GaAs/GaAlAs pour différentes valeurs du champ de grille : $E_{\|}=1 \mathrm{kV} / \mathrm{cm}, 4_{\mathrm{L}}:$ densité d'électrons en vallée $\mathrm{L}$.

[Carrier density in GaAs/GaAlAs TEGFET's for different electric fields at the gate.]

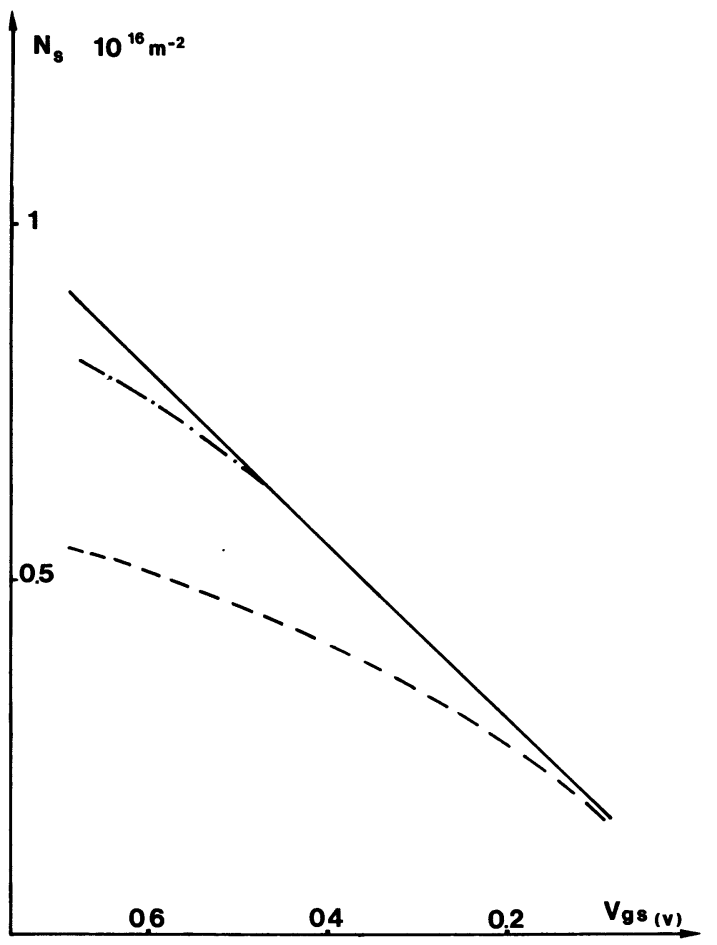

Fig. 7. - Charge superficielle $N_{\mathrm{s}}$ en fonction de la tension $V_{\text {gs }}$ dans le TEGFET GaAs/GaAlAs : (—) $E_{\|}=$ $\left.0,1 \mathrm{kV} / \mathrm{cm} ;(--\longrightarrow) E_{\|}=1 \mathrm{kV} / \mathrm{cm} ;(---)\right) E_{\|}=10 \mathrm{kV} / \mathrm{cm}$.

[Sheet concentration versus gate voltage in the GaAs/ GaAlAs TEGFET's.]

\section{Modèle théorique du TEGFET.}

Notre modèle théorique du TEGFET est dérivé de celui qui a été développé au laboratoire pour étudier les TEC conventionnels [5]. Il repose (Fig. 8) sur un 


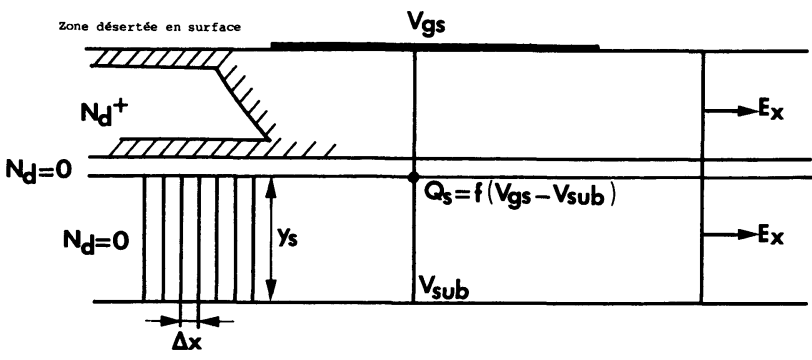

Fig. 8. - Principe de la modélisation.

[Principle of the model.]

certain nombre d'hypothèses simplificatrices qui permettent d'éviter une résolution entièrement bidimensionnelle de la structure, résolution qui nécessiterait des temps calculs et des espaces mémoires très importants à cause des caractéristiques géométriques et technologiques particulières de cette structure.

Le rapport $L_{\mathrm{g}} / a$ des structures étudiées est toujours beaucoup plus grand que 1 et une approche quasi unidimensionnelle est alors possible.

Conformément à l'étude précédente et à des résultats expérimentaux [6], la dynamique électronique dans GaAs est supposée être identique à celle du matériau non dopé en volume. De plus le caractère non stationnaire du transit des électrons sous la grille sera pris en compte par la méthode des équations de conservation du moment et de l'énergie. Dans AlGaAs, la vitesse sera par contre considérée comme uniquement fonction du champ électrique.

La quantité de porteurs dans la couche d'accumulation ne dépend que de la différence de potentiel entre le canal conducteur et la grille.

Cette quantité de charge accumulée est alors calculée en utilisant un modèle unidimensionnel de l'hétérojonction qui tient compte de la dégénérescence des électrons et des phénomènes de quantification $[4,7]$.

Le modèle repose alors sur la résolution, à chaque abscisse de l'axe source-drain discrétisé, des quatre équations suivantes :

$$
\begin{aligned}
& I_{\mathrm{ds}}=q Z\left(Q_{0} v_{0 x}+Q_{1} v_{1 x}\right) \\
& \frac{\partial E_{x}}{\partial x}+\frac{\partial E_{y}}{\partial y}=\frac{q}{\varepsilon}\left(n-N_{\mathrm{d}}\right) \\
& \frac{\mathrm{d} \bar{\varepsilon}}{\mathrm{d} x}=q E_{x}-\frac{\bar{\varepsilon}-\varepsilon_{0}}{v_{0 x} \cdot \tau_{\varepsilon}} \\
& 0=q E_{x}-m^{*} v_{0 x} \cdot \frac{\mathrm{d} v_{0 x}}{\mathrm{~d} x}-\frac{m^{*} v_{0 x}}{\tau_{\mathrm{m}}} .
\end{aligned}
$$

Dans ces expressions, $I_{\mathrm{ds}}$ représente le courant drain, $Q_{0}$ et $Q_{1}$ les quantités de porteurs dans GaAs et GaAlAs respectivement, $v_{0 x}$ et $v_{1 x}$ les vitesses moyennes longitudinales dans ces deux matériaux, $\bar{\varepsilon}$ l'énergie moyenne des porteurs dans GaAs, $m^{*}$ la masse efficace, $\tau_{\mathrm{m}}$ et $\tau_{\varepsilon}$ les temps de relaxation du moment et de l'énergie.
La méthode de résolution de ces équations est identique à celle qui a été mise au point pour le TEC classique [5].

Pour un courant drain et un potentiel de grille fixés, le modèle permet d'obtenir les évolutions de la vitesse $v_{0 x}$, de l'énergie $\bar{\varepsilon}$, du champ longitudinal $E_{x}$ et des quantités de porteurs dans chacun des matériaux le long de l'axe source-drain. Il est alors possible de calculer la tension $V_{\mathrm{ds}}$ ainsi que la quantité de charge emmagasinée dans le composant.

Autour d'un point de fonctionnement donné, de petites variations $\Delta I_{\mathrm{ds}}$ et $\Delta V_{\mathrm{gs}}$ permettent d'obtenir les principaux éléments du schéma équivalent $: g_{\mathrm{m}}$, $g_{\mathrm{d}}, C_{\mathrm{gs}}, C_{\mathrm{gd}}, \tau$ et $R_{\mathrm{i}}$. Notons que la prise en compte d'une certaine épaisseur de GaAs permet d'obtenir, bien que le modèle soit quasi unidimensionnel, une valeur non nulle de la conductance de sortie permettant alors une détermination du gain en puissance. Signalons enfin qu'une version de ce programme permettant l'étude du bruit est en cours d'élaboration.

Afin de tester la validité du modèle théorique, nous en avons comparé les résultats avec l'expérience. Un exemple typique de comparaison est donné figure 9 où est représentée l'évolution de la transconductance en fonction de la tension grille $V_{\mathrm{gs}}$.

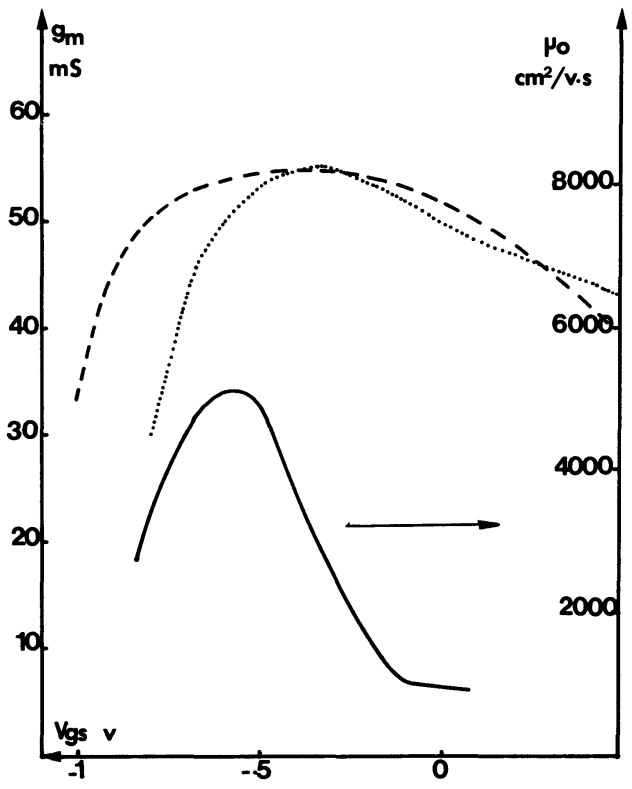

Fig. 9. - Comparaison des transconductances théorique et expérimentale et profil de mobilité en fonction de la tension grille source. (------) théorie ;(......) expérience ; (-) mobilité.

[Comparison between theoretical and experimental transconductances and mobility profile as a function of the gate voltage.]

Jusqu'à $V_{\mathbf{g s}}=-0,5 \mathrm{~V}$, l'accord est satisfaisant, puis les deux courbes diffèrent assez significativement, la transconductance expérimentale chutant plus rapidement. 
La raison de ce désaccord est que la mobilité expérimentale (mesurée par magnéto-résistance) également représentée sur la figure 9 dépend fortement de la tension $V_{\mathrm{gs}}$. Par contre, cette mobilité est supposée indépendante de $V_{\mathrm{gs}}\left(\mu_{0}=4500 \mathrm{~cm}^{2} / \mathrm{Vs}\right)$ dans le modèle théorique, ceci par souci de simplicité.

Ainsi notre modèle théorique permet d'obtenir des ordres de grandeurs corrects alors qu'il ne possède aucun paramètre ajustable. En effet, les grandeurs introduites dans le modèle telles que $R_{\mathrm{s}}, N_{\mathrm{d}}, L_{\mathrm{g}}, a$, etc... ont été mesurées sur le composant ou données par le constructeur.

Nous avons effectué la comparaison sur la transconductance car celle-ci présente un grand intérêt pour l'étude de la dynamique des électrons. En effet, après la correction due à la résistance de source, la transconductance intrinsèque peut s'écrire [7] :

$$
g_{\mathrm{m}_{0}}=\frac{\varepsilon \cdot Z \cdot \bar{v}}{a+80 \AA} .
$$

La mesure de la transconductance représente donc une mesure indirecte de la vitesse moyenne des électrons.

Nous avons utilisé cette propriété pour étudier l'évolution de la vitesse moyenne des porteurs sous la grille en fonction de la température, et par conséquent en fonction de la mobilité en champ faible. La figure 10 montre que cette vitesse moyenne croît sensiblement lorsque la température diminue. A $77 \mathrm{~K}$, la transconductance, mesurée à partir des caractéristiques continues $I_{\mathrm{ds}}\left(V_{\mathrm{gs}}, V_{\mathrm{ds}}\right)$, présente une valeur particulièrement élevée de $540 \mathrm{mS} / \mathrm{mm}$. Cette valeur est à notre connaissance la valeur la plus élevée publiée à ce jour.

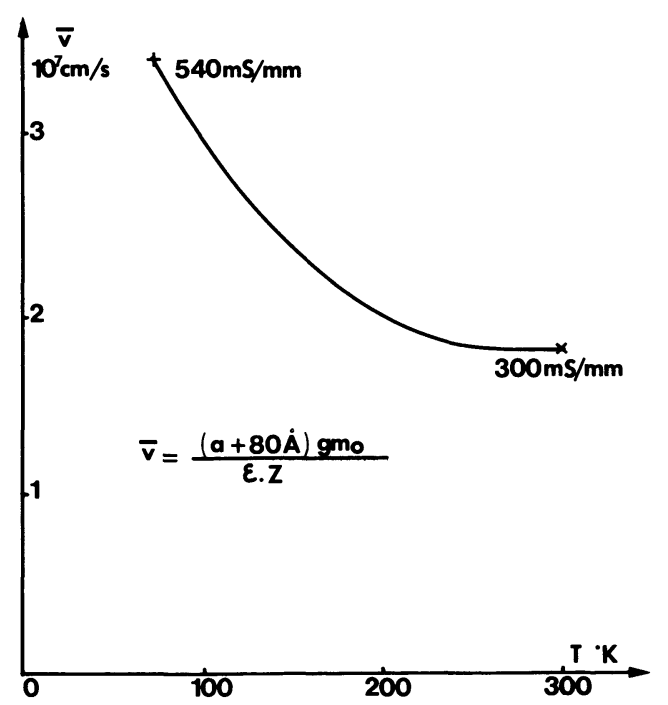

Fig. 10. - Evolution expérimentale de la vitesse moyenne des porteurs sous la grille en fonction de la température.

[Experimental evolution of the average carrier velocity under the gate as a function of the temperature.]
Remarque : avec une transconductance aussi élevée, on pouvait s'attendre à de très bonnes performances hyperfréquences en basse température. En fait, la transconductance diminue rapidement avec la fréquence. Les raisons de cette décroissance ne sont pas encore bien comprises, et ce phénomène doit être étudié avec précision afin de déterminer s'il est intrinsèque à l'hétérojonction ou s'il n'est dû qu'à un effet parasite qui pourra être éliminé.

\section{Performances comparées des TEGFET et des TEC classiques.}

Le modèle théorique que nous venons de décrire permet d'étudier les performances des TEGFET en amplification micro-ondes et en logique à couplage direct.

4. 1 L'AMPLIFICATION MICRO-ONDES. - Notre étude a principalement porté sur le gain en puissance. Si l'on néglige les principaux éléments parasites tels que les résistances $\boldsymbol{R}_{\mathrm{s}}$ et $\boldsymbol{R}_{\mathrm{g}}$ et l'inductance de source $L_{\mathrm{s}}$, le gain en puissance peut s'écrire :

$$
G=\left(\frac{f_{\mathrm{c}}}{f}\right)^{2} \cdot \frac{1}{4 R_{\mathrm{i}}\left(g_{\mathrm{d}}+\pi f_{\mathrm{c}} C_{\mathrm{gd}}\right)}
$$

Nous avons représenté (Fig. 11) l'évolution de ce gain en puissance intrinsèque, ainsi que la fréquence pour laquelle ce gain devient unité en fonction de la longueur de grille. Nous constatons une supériorité du TEGFET sur le TEC classique d'environ $4 \mathrm{~dB}$. D'autre part, la fréquence de coupure de ce gain intrinsèque est très élevée puisqu'elle atteint $500 \mathrm{GHz}$ pour une longueur de grille de $0,2 \mu$.

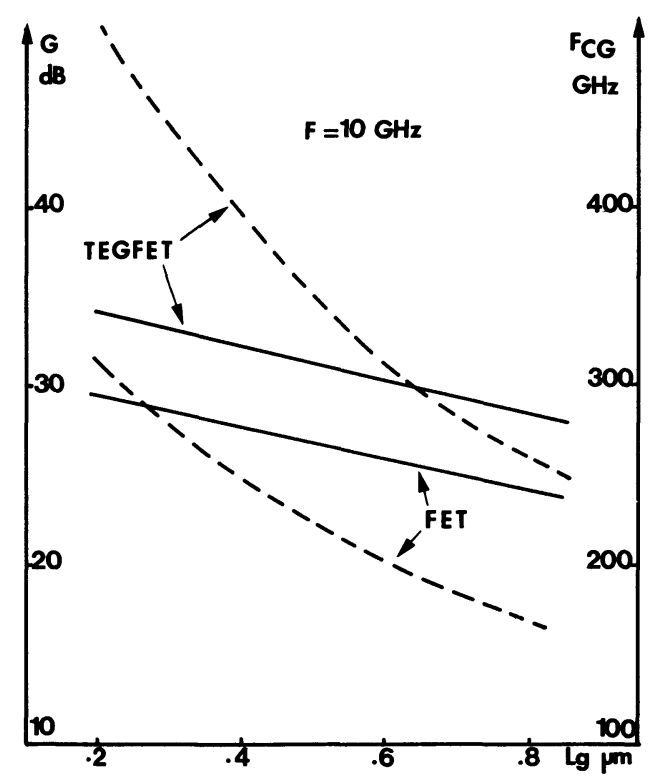

Fig. 11. - Evolutions du gain intrinsèque en puissance disponible et de la fréquence de coupure de ce gain en fonction de la longueur de grille. $T=300 \mathrm{~K}:(-)$ gain; (------) fréquence de coupure du gain $F_{\mathrm{cg}}$.

[Evolution of the intrinsic MAG and cut-off frequency as a function of the gate length.] 
Cette supériorité est d'ailleurs confirmée par l'expérience; à $10 \mathrm{GHz}$, un facteur de bruit de $1,3 \mathrm{~dB}$ et un gain associé de $12 \mathrm{~dB}$ ont été mesurés [8] sur un TEGFET de longueur de grille $0,5-0,6 \mu$. Cette performance peut être comparée favorablement avec celle des meilleurs TEC classiques alors que les éléments parasites du TEGFET, principalement la résistance de source, demeurent plus élevés.

Cette supériorité provient essentiellement de la fréquence de coupure intrinsèque $g_{\mathrm{m}_{0}} / 2 \pi C_{\mathrm{gs}}$. Nous avons représenté l'évolution de cette fréquence $f_{\mathrm{c}}$ en fonction de la longueur de grille sur la figure 12 . Plus la grille est courte, plus l'écart se creuse entre le TEC classique et le TEGFET. Ce phénomène est dû à une exaltation du phénomène de survitesse pour les grilles courtes et pour les mobilités élevées. La vitesse moyenne des électrons sous la grille, également représentée sur la figure 12, présente en effet, une valeur plus élevée dans le cas du TEGFET.

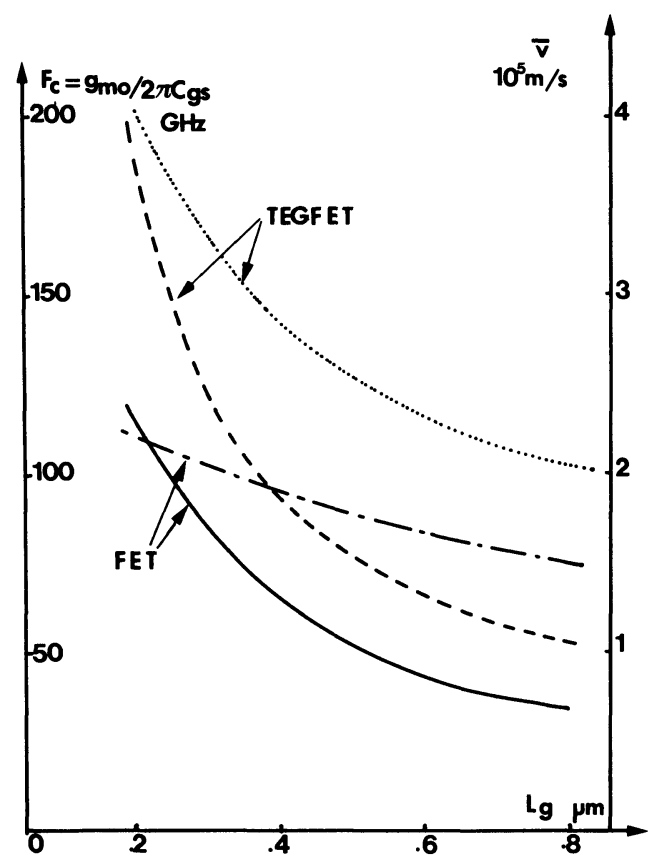

Fig. 12. - Evolution de la fréquence de coupure $g_{\mathrm{m}_{0}} / 2 \pi C_{\mathrm{gs}}$ et de la vitesse moyenne en fonction de la longueur de grille $T=300 \mathrm{~K}$ :

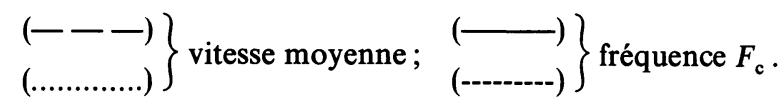

[Evolution of the cut-off frequency $g_{\mathrm{m}_{0}} / 2 \pi C_{\mathrm{gs}}$ and the average velocity as a function of the gate length.]

4.2 LeS CIRCUITS LOGIQUeS À COUPLAGE DIRECT. Expérimentalement, de très bonnes performances ont été également obtenues en logique DCFL : des temps de propagation de l'ordre de 17 ps pour des oscillateurs en anneau fonctionnant à température ambiante [9].

Pour calculer le temps de propagation des portes DCFL, nous utilisons un modèle dérivé de celui de Ino et al. $[10,11]$. Durant les processus de charge et de décharge, le point de fonctionnement du système suit le cycle A.B.A. représenté sur la figure 13. Le temps de propagation peut donc s'écrire :

$$
T_{\mathrm{pd}}=\frac{Q_{\mathrm{B}}-Q_{\mathrm{A}}+C_{\mathrm{p}} \Delta V}{4}\left(\left\langle\frac{1}{I_{\mathrm{ch}}}\right\rangle+\left\langle\frac{1}{I_{\mathrm{dech}}}\right\rangle\right) .
$$

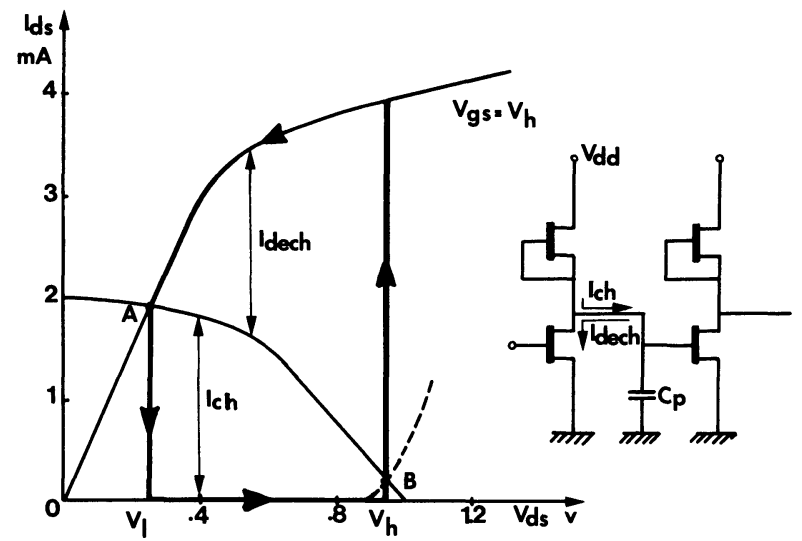

Fig. 13. - Cycle de fonctionnement d'un inverseur DCFL.

[Switching diagram for a DCFL inverter.]

Dans cette expression $Q_{\mathrm{B}}$ et $Q_{\mathrm{A}}$ sont les charges emmagasinées par un transistor pour des potentiels de grille bas et haut, $C_{\mathrm{p}}$ la capacité parasite inter-étage, $\Delta V$ l'excursion logique, $I_{\mathrm{ch}}$ et $I_{\text {dech }}$ les courants de charge et de décharge, les symboles $\langle>$ représentant la moyenne prise entre $10 \%$ et $90 \%$ de l'excursion logique.

Nous avons représenté sur la figure 14 les évolu-

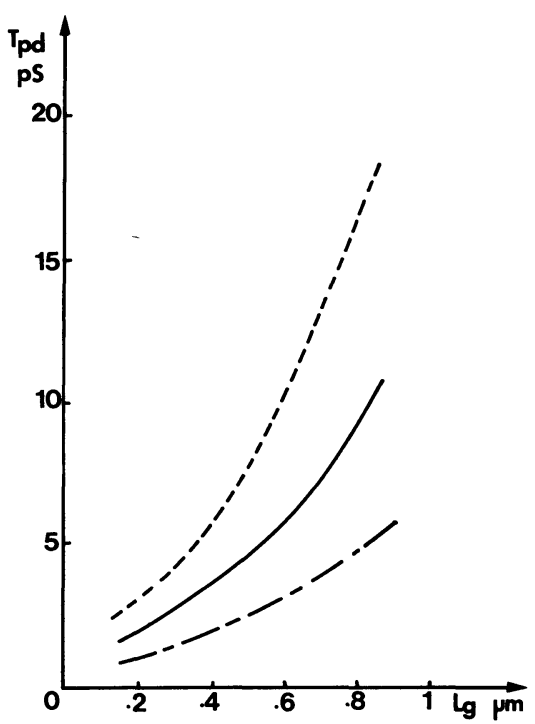

Fig. 14. - Evolution du temps de propagation intrinsèque en fonction de la longueur de grille. (-------) FET $T=300 \mathrm{~K}$; (-) TEGFET $T=300 \mathrm{~K}$; (-. - - ) TEGFET $T=77 \mathrm{~K}$

[Evolution of the intrinsic propagation delay time as a function of the gate length.] 
tions du temps de propagation intrinsèque $\left(C_{\mathrm{p}}=0\right)$ en fonction de la longueur de grille.

Nous constatons, que le temps de propagation intrinsèque du TEGFET est inférieur à ceux du TEC classique et qu'il décroît sensiblement lorsque la température diminue. Ces effets s'expliquent de nouveau grâce à des considérations dynamiques. Nous avons représenté sur la figure 15, l'évolution de la vitesse des porteurs sous la grille pour un TEC classique et pour un TEGFET à $300 \mathrm{~K}$ et $77 \mathrm{~K}$. Dans le cas d'un TEC classique, la mobilité n'évoluant pratiquement pas lorsque la température diminue, une évolution identique doit être observée à $77 \mathrm{~K}$.

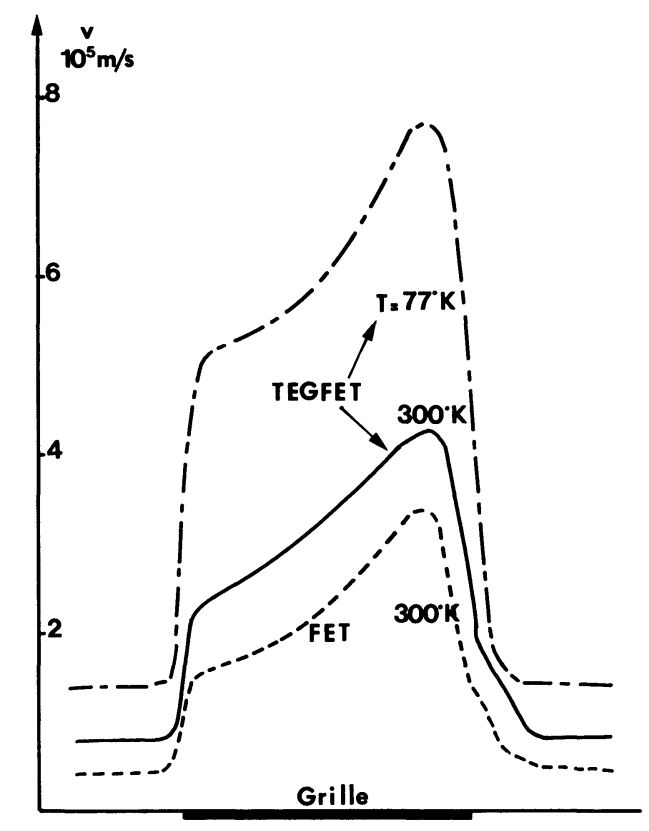

Fig. 15. - Evolution de la vitesse des porteurs sous la grille (---) FET $T=300 \mathrm{~K}$; (—) TEGFET $T=300 \mathrm{~K}$; (-. - - ) TEGFET $T=77 \mathrm{~K}$.

[Evolution of the carrier velocity under the gate.]

La vitesse des porteurs étant plus élevée dans le cas des TEGFET, les courants de charge $I_{\mathrm{ch}}$ et de décharge $I_{\text {dech }}$, sont donc plus importants. Pour un même transfert de charge $Q_{\mathrm{B}}-Q_{\mathrm{A}}$, le TEGFET sera donc plus rapide que le TEC classique.

Néanmoins, la plus grande part du temps de propagation d'une part logique provient non pas du transistor inverseur lui-même mais des différentes capacités parasites. Nous avons donc étudié l'influence de la capacité parasite interétage $C_{\mathrm{p}}$.

Le temps de propagation $T_{p d}$ extrinsèque est représenté sur la figure 16 en fonction de la valeur de la capacité parasite $C_{\mathrm{p}}$.

Nous pouvons noter que la supériorité du TEGFET est conservée et qu'elle s'accroît même lorsque $C_{\mathrm{p}}$ augmente. Cet effet particulier peut s'expliquer grâce à l'expression du temps de propagation. Nous constatons que les courants $I_{\text {ch }}$ et $I_{\text {dech }}$ interviennent comme coefficient multiplicatif que l'on tienne compte

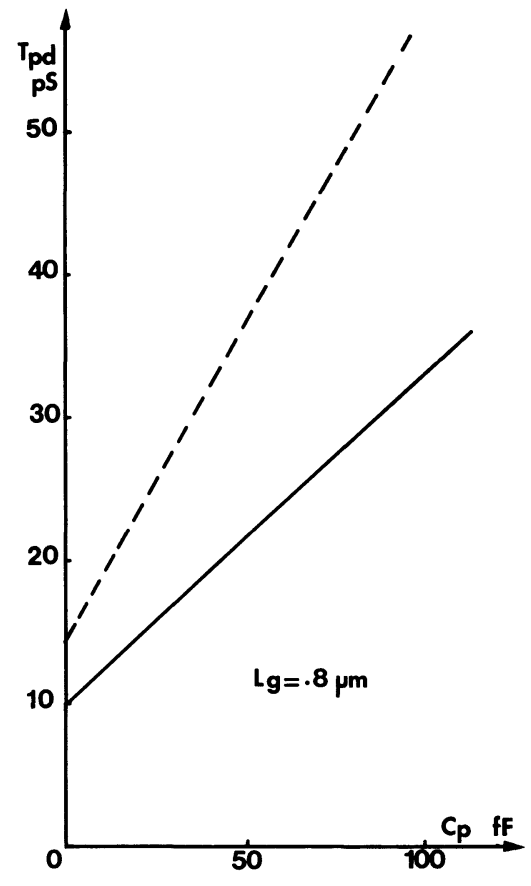

Fig. 16. - Evolution du temps de propagation $T_{\text {pd }}$ en fonction de la capacité parasite inter-étage. $T=300 \mathrm{~K}$, (-----) FET ; ( $\longrightarrow$ TEGFET.

[Evolution of the propagation delay time as a function of the parasitic interstage capacitance.]

ou non des capacités parasites. La différence entre TEC et TEGFET étant essentiellement d'origine dynamique, celle-ci se retrouve essentiellement sur les courants de charge $I_{\text {ch }}$ et décharge $I_{\text {dech }}$ et sera conservée malgré l'existence des capacités parasites.

\section{Conclusion.}

L'utilisation de la procédure Monte-Carlo nous a permis d'étudier la dynamique des électrons dans une couche d'accumulation, le phénomène de transfert dans l'espace réel, et l'influence de la tension grille sur ce transfert a été mis en évidence. Les caractéristiques $v\left(E_{\|}\right)$obtenues présentent des mobilités en champ faible très voisines du matériau intrinsèque en volume. Par contre, les vitesses de saturation diffèrent du fait du transfert des électrons dans leur matériau d'origine aux propriétés dynamiques différentes.

Ces résultats nous ont permis de mettre au point un modèle théorique du TEGFET. Une étude comparative des FET classiques et des TEGFET a été menée, qui montre la supériorité intrinsèque du TEGFET. Celle-ci peut être attribuée aux vitesses plus élevées que peuvent atteindre les électrons dans un matériau non dopé. Les gains que l'on peut espérer obtenir sont donc plus importants, et les temps de propagation des portes logiques plus courts.

Pour que cette supériorité se concrétise en pratique, les éléments parasites, en particulier $R_{\mathrm{s}}$, doivent 
être faibles. Par contre, l'effet de la capacité interétage $C_{\mathrm{p}}$ dans les circuits logiques DCFL ne doit pas remettre en cause les meilleures performances potentielles des TEGFET.

\section{Remerciements.}

Nous remercions N. T. Linh et ses collaborateurs du laboratoire central de recherches de Thomson C.S.F. pour la fourniture des échantillons.

\section{Bibliographie}

[1] Mimura, T., Hiyamizu, S., Joshen, K., Hikosaka, K., « Enhancement mode high electron mobility transistors for logic applications ", Jpn. J. Appl. Phys. 20 (1981) L317.

[2] Delagebeaudeuf, D., Delecluse, P., Etienne, P., Laviron, M., Chaplart, J., Linh, N. T., « Two dimensional electron gas Mesfet structure ", Electron. Lett. 16, $\mathrm{n}^{\circ} 17$ (1980) 667.

[3] Drummond, T. J., Kopp, W., Thorne, R. E., Fischer, R. and Morkoc, H., "Influence of AlGaAs buffer layers on the performances of modulation doped field effect transistors ", Appl. Phys. Lett. 40 (1982) 879.

[4] Delagebeaudeuf, D. and Linh, N. T., « Metal n-AlGaAs-GaAs two dimensional electron gas FET », I.E.E.E. Trans. 29 (1982) 950-955.

[5] CAPPY, A., "Sur un nouveau modèle de transistor à effet de champ à grille submicronique ", Thèse 3 e cycle, Lille, 1981.

[6] Drummond, T. J., KopP, W., Morkoc, H., KeEver, M., " Transport in modulation doped structures $\mathrm{AlGaAs} / \mathrm{GaAs}$ and correlations with Monte-Carlo calculations GaAs " Appl. Phys. Lett. 41 (3) (1982) 277-279.
[7] Lee, K., Shur, M. S., Drummond, T. J. and Morkoc, H., "Current voltage and capacitance voltage characteristics of modulation doped field effect transistors ", I.E.E.E. Trans. 30, no 3 (1983), 207-212.

[8] Delecluse, P., Delagebeaudeuf, D., Laviron, M., Tung, P. N., Chevrier, J., Linh, N. T., « TEGFET par EJM : récents développements ", Journées Nationales semiconducteurs composés III-V, Toulouse, juillet 1983.

[9] Tung, P. N., Delagebeaudeuf, D., Laviron, M., Delecluse, P., Chaplart, J., Linh, N. T., « High speed two dimensional electron gas FET logic ", Electron. Lett. 18 (1982) 109-110.

[10] Ino, M., Hirayama, M. and Ohmori, M., " Analysis for optimum threshold voltage and load current of E-D type GaAs DCFL circuits " Electron. Lett. 17 (1981) 522-523.

[11] Togashi, M., Ino, M. and Hirayama, M., « Sub. FJ inverter analysis for GaAs VLSI ", Electron. Lett. 19 (1983), 231-232. 